\title{
A NOTE ON THE INTERRELATION OF SUBSETS OF INDEPENDENT VARIABLES OF A CONTINUOUS FUNCTION WITH CONTINUOUS FIRST DERIVATIVES ${ }^{1}$
}

\section{WASSILY LEONTIEF}

Let $X$ be a set of $n$ variables $x_{1}, x_{2}, \cdots, x_{n}$ and $y$ a continuous function of these variables, $y=f\left(x_{1}, x_{2}, \cdots, x_{n}\right)$, also written as $f(X)$ with continuous partial derivatives of the first, second, and third orders. Throughout the following discussion all the first partial derivatives of $f\left(x_{1}, x_{2}, \cdots, x_{n}\right)$ are assumed to be different from zero.

Let $S$ be a proper subset of $X$ and $S^{\prime}$ the subset which is complementary to $S$ in $X$.

Definition I. A subset $S$ of independent variables is locally functionally separable at a point $\left(a_{1}, a_{2}, \cdots, a_{n}\right)$ within the set $X$ in $y=f(X)$ if there exist some function $\phi$ with continuous first derivatives and defined in some neighborhood of $\left(a_{1}, a_{2}, \cdots, a_{v}\right)$ and another function $\psi$ also with continuous first derivatives and defined in some neighborhood of $\left(b, a_{v+1}, a_{v+2}, \cdots, a_{n}\right)$ where $b=\phi\left(a_{1}, a_{2}, \cdots, a_{v}\right)$ such that

$$
y=f(X)=\psi\left(\phi(S), S^{\prime}\right) .
$$

The function $\phi(S)$ is locally separable in $f(X)$.

From the definition of a locally separable subset it follows that each of the original variables, that is, each of the elements of $X$, is a locally separable subset of $X$ in $f(X)$; so is also the set $X$ itself.

Let ${ }_{i j} R$ be defined by

$$
{ }_{i j} R=f_{i}^{\prime} / f_{j}^{\prime}
$$

where $f_{i}^{\prime}$ and $f_{i}^{\prime}$ are the partial derivatives of $f\left(x_{1}, x_{2}, \cdots, x_{n}\right)$ with respect to $x_{i}$ and $x_{j}$. The necessary and sufficient condition for ${ }_{i j} R$ to be independent of $x_{k}$ is

$$
{ }_{i j} R_{k}^{\prime}=\frac{f_{i k}^{\prime \prime} f_{i}^{\prime}-f_{j k}^{\prime \prime} f_{i}^{\prime}}{\left[f_{i}^{\prime}\right]^{2}}=0
$$

Received by the editors June 11, 1946, and, in revised form, October 9, 1946.

1 The subject of this note suggested itself by a study of "production functions" and "consumption functions" in mathematical economics.

The problem considered in this paper bears some relation to the XIIIth problem of Hilbert: D. Hilbert, Nach. Ges. Wiss. Göttingen (1900) p. 280; cf. also Pólya and Szegö, Aufgaben und Lehrsätze aus der Analysis, vol. I, problems 119 and 119a, pp. 61-62 and 220-222. 
where $f_{i \mathbf{k}}^{\prime \prime}$ and $f_{j \mathbf{k}}^{\prime \prime}$ are the second partial derivatives of $f\left(x_{1}, x_{2}, \cdots, x_{n}\right)$. Thus if ${ }_{i j} R_{k}^{\prime}=0$ then ${ }_{j i} R_{k}^{\prime}=0$.

Since

$$
{ }_{j l} R_{k}^{\prime} f_{l}^{\prime} f_{i}^{\prime}={ }_{i l} R_{k}^{\prime} f_{l}^{\prime} f_{j}^{\prime}-{ }_{i j} R_{k}^{\prime}\left[f_{j}^{\prime}\right]^{2},
$$

if ${ }_{i l} R$ and ${ }_{i j} R$ are independent of $x_{k},{ }_{j l} R$ is also independent of $x_{k}$.

Similarly the relationship

$$
{ }_{i k} R_{j}^{\prime}-{ }_{j k} R_{i}^{\prime}={ }_{i j} R_{k}^{\prime}\left[f_{j}^{\prime} / f_{k}^{\prime}\right]^{2}
$$

implies that if ${ }_{i k} R$ is independent of $x_{j}$ and ${ }_{j k} R$ is independent of $x_{i}$ then ${ }_{i j} R$ is independent of $x_{k}$.

Theorem I. A subset $S$ is locally functionally separable from $X$ in $f(X)$ if and only if the following set of equations is satisfied $:_{s_{i} s_{j}} R_{s^{\prime}}^{\prime}=0$. The first two subscripts to $R$ refer to any two identical or different elements in the subset $S$, while the last subscript indicates any element of the subset $S^{\prime}$ which is complementary to $S$ in $X$.

Let $S$ be a locally separable subset, that is, relationship (1) is known to be true. If $x_{i}$ and $x_{j}$ are elements in $S$ and $x_{k}$ is an element in $S^{\prime}$, then ${ }_{i j} R=f_{i}^{\prime} / f_{j}^{\prime}=\phi_{i}^{\prime}(S) / \phi_{j}^{\prime}(S)$ and consequently ${ }_{i j} R_{k}^{\prime}=0$.

In order to show that the local separability condition stated above is not only necessary but also sufficient let the function $\phi(S)$ be defined by

$$
\phi(S)=f\left(S, S^{\prime o}\right),
$$

where $f\left(S, S^{o}\right)$ is obtained from $f(X)$ by assigning to each of the $n-v$ elements $x_{v+1}, x_{v+2}, \cdots, x_{n}$ of the subset $S^{\prime}$ a constant value $x_{v+1}^{0}, x_{v+2}^{0}, \cdots, x_{n}^{0}$.

The subset $S$ comprising the elements $x_{1}, x_{2}, \cdots, x_{v}$ is locally functionally separable in $X$ if the function $f(X)$ can be shown to be expressed in terms of $f\left(S, S^{\prime o}\right)$ and of the set of the $n-v$ variables $x_{v+1}, x_{v+2}, \cdots, x_{n}$. Let these be considered as parameters in $f(X)$. The derivatives of the functions $f(X)$ and $f\left(S, S^{\prime o}\right)$, with respect to the $v$ variables $x_{1}, x_{2}, \cdots, x_{v}$, form a matrix

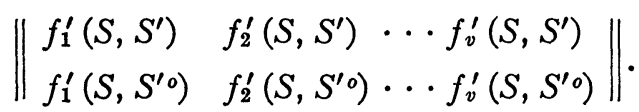

All the terms of the first row of this matrix are proportional to the corresponding terms of the second row if the relationship

$$
f_{i}^{\prime}\left(S, S^{\prime o}\right) / f_{j}^{\prime}\left(S, S^{\prime o}\right)=f_{i}^{\prime}\left(S, S^{\prime}\right) / f_{j}^{\prime}\left(S, S^{\prime}\right)
$$

holds for all $i, j \leqq v$. It obviously holds if $S^{\prime}=S^{\prime o}$, that is, in the special 
case when the parameters $x_{v+1}, x_{v+2}, \cdots, x_{n}$, which are elements of $S^{\prime}$, are assigned the particular values $x_{v+1}^{0}, x_{v+2}^{0}, \cdots, x_{v}^{0}$. But if ${ }_{i j} R_{k}^{\prime}=0$ for all $i, j \leqq v$ and $k>v$, that is, if the last term of the equation above is independent of the variables comprised in the subset $S^{\prime}$, this equation holds also if $x_{v+1}, x_{v+2}, \cdots, x_{n}$ acquire values other than $x_{v+1}^{0}, x_{v+2}^{0}, \cdots, x_{n}^{0}$. In this case the two rows of the matrix are linearly dependent: the first row equals the second multiplied by $f_{1}^{\prime}\left(S, S^{\prime}\right) / f_{1}^{\prime}\left(S, S^{\prime o}\right)$. Since, furthermore, the element $f_{v}^{\prime}\left(S, S^{\prime o}\right)$ located in the lower right-hand corner of the matrix is by assumption different from zero, $f(X)$ can, according to the general theorem on functional dependence, be expressed in the form $f(X)=\psi\left(f\left(S, S^{\prime o}\right), S^{\prime}\right)$, where $\psi$ is a function having continuous first, second, and third partial derivatives, or if we substitute $\phi(S)$ for $f\left(S, S^{\prime o}\right)$

$$
f(X)=\psi\left(\phi(S), S^{\prime}\right)
$$

which shows that the condition of local separability of subset $S$ in $X$ as stated in Theorem $I$ is a sufficient one.

The first half of the foregoing argument holds also if the function $\phi(S)$ by which $S$ is locally separable from $X$ in $f(X)$ is more generally defined as $\phi(S)=\Phi\left(f\left(S, S^{\prime o}\right)\right)$ where $\Phi$ is some continuous function with locally nonvanishing first derivatives.

The conditions of simultaneous local separability of mutually exclusive locally separable subsets of any given set of independent variables can be stated as follows :

LEMma. Given $A, B, C$, three mutually exclusive subsets of set $X$ and $G$ the rest of $X$, if each of the sets $A, B, C$ is locally functionally separable from $X$ in $f(X)$, these subsets can also be separated simultaneously from $X$ in $f(X)$.

Proceeding along the line of argument used in proving Theorem I, let the individually locally separable functions $\alpha(A), \beta(B), \gamma(C)$ be given the particular forms $f\left(A, B^{o}, C^{o}, G^{o}\right), f\left(A^{\circ}, B, C^{o}, G^{o}\right)$ and $f\left(A^{\circ}, B^{o}, C, G^{o}\right)$. Differentiating $f(X)$ and the three separable functions with respect to all the variables comprised in subsets $A, B$, and $C$, form the four-rowed matrix:

$$
\left\|\begin{array}{ccccccc}
f_{a_{1}}^{\prime}(A, B, C, G) & \cdots & f_{b_{1}}^{\prime}(A, B, C, G) & \cdots & f_{c_{1}}^{\prime}(A, B, C, G) & \cdots & f_{c_{v}}^{\prime}(A, B, C, G) \\
f_{a_{1}}^{\prime}\left(A, B^{o}, C^{o}, G^{o}\right) & \cdots & 0 & \cdots & 0 & \cdots & 0 \\
0 & \cdots & f_{b_{1}}^{\prime}\left(A^{o}, B, C^{o}, G^{o}\right) & \cdots & 0 & \cdots & 0 \\
0 & \cdots & 0 & \cdots & f_{c_{1}}^{\prime}\left(A^{o}, B^{o}, C, G^{o}\right) & \cdots & f_{c_{v}}^{\prime}\left(A^{o}, B^{o}, C, G^{o}\right)
\end{array}\right\| .
$$

The first $m$ columns of this matrix contain the derivatives with re- 
spect to the $m$ variables $a_{1}, a_{2}, \cdots, a_{m}$ contained in the subset $A$, the next $r$ columns consist of derivatives with respect to the $r$ variables $b_{1}, b_{2}, \cdots, b_{r}$ included in subset $B$ and the last $v$ columns are composed of the derivatives with respect to the $v$ elements of subset $C$. All elements of subset $G$ appearing under the function signs in row one are regarded as parameters. An argument similar to that presented in the proof of Theorem I shows that the four rows of the matrix are linearly dependent. Since all derivatives are assumed to be different from zero, the determinant formed by the second, third, and fourth elements of the first, second, and third columns (as written out above) respectively is also different from zero: the rank of the matrix is three. From these two properties of the matrix it follows that $f(X)$ is a function of $f\left(A, B^{o}, C^{o}, G^{o}\right), f\left(A^{o}, B, C^{o}, G^{o}\right), f\left(A \bullet, B^{o}, C, G^{o}\right)$ and of the variables comprized in the subset $G$. In general:

$$
f(X)=\Phi(\alpha(A), \beta(B), \gamma(C), G) .
$$

THEOREM II. (a) If two functionally locally separable subsets $S$ and $T$ of $X$ in $f(X)$, of which neither is a subset of the other, intersect each other, then each of the three nonoverlapping sets-one of which comprises all elements of $S$ which are not elements of $T$, the second consists of all the elements of $T$ which are not elements of $S$, and the third contains all elements common to $S$ and $T$-is also a functionally locally separable subset of $X$ in $f(X)$. (b) Furthermore if $\phi(S \cup T)$ is a function of $S \cup T$ locally separable in $f(X)$ the three nonoverlapping sets mentioned above are additively locally separable subsets of $S \cup T$ in $\phi(S \cup T)$; that is, if $A, B$, and $C$ are these three subsets, there exist functions $\alpha, \beta$, and $\gamma$ with continuous first derivatives such that

$$
\phi(S \cup T)=\alpha(A)+\beta(B)+\gamma(C) .
$$

Let $X$ be partitioned in four nonoverlapping subsets $A, B, C$, and $G$,

$$
X=A \cup B \cup C \cup G,
$$

and the two overlapping subsets defined as

$$
S=A \cup B, \quad T=C \cup B .
$$

$S$ and $T$ are locally separable from $X$ in $f(X)$, that is,

$$
\begin{aligned}
& f(X)=v(\phi(A \cup B), C \cup G), \\
& f(X)=w(\theta(C \cup B), A \cup G) .
\end{aligned}
$$

First let it be shown that these two equations imply 


$$
f(X)=u(\alpha(A), \beta(B), \gamma(C), G) .
$$

From (4) and (5) it follows, according to Theorem I, that

$$
\begin{aligned}
& { }_{a b} R_{c}^{\prime}=0, \quad{ }_{a a} R_{c}^{\prime}=0, \quad{ }_{b b} R_{c}^{\prime}=0, \\
& { }_{a b} R_{o}^{\prime}=0, \quad{ }_{a a} R_{b}^{\prime}=0, \quad{ }_{b b} R_{\sigma}^{\prime}=0, \\
& { }_{c b} R_{a}^{\prime}=0, \quad{ }_{c c} R_{a}^{\prime}=0, \quad{ }_{b b} R_{a}^{\prime}=0, \\
& { }_{c b} R_{a}^{\prime}=0, \quad{ }_{c c} R_{a}^{\prime}=0, \quad{ }_{b b} R_{o}^{\prime}=0,
\end{aligned}
$$

where $a, b, c$, and $g$ represent any elements in $A, B, C$, and $G$ respectively, while the combinations of two identical subscripts such as $a a$ or $b b$ refer to any two elements, identical or different, both belonging to the same set, in this instance $A$ or $B$ respectively.

From ${ }_{a b} R_{c}^{\prime}=0$ and ${ }_{c b} R_{a}^{\prime}=0$ we have, by (3), ${ }_{a c} R_{b}^{\prime}=0$. Let the subscript $c_{1}$ refer to one particular element in $C$ and $b_{k}$ to one particular element in $B$, then on the basis of the last relationship $a_{a_{1} c_{1}} R_{b_{k}}^{\prime}=a_{a_{2} c_{1}} R_{b_{k}}^{\prime}$ $=\cdots=a_{a_{m} c_{1}} R_{b_{k}}^{\prime}=0$, where $a_{1}, a_{2}, \cdots, a_{m}$ refer to all the $m$ elements of $A$. Applying to these $m$ equations the rule derived from (2) we have $a_{i} a_{j} R_{b_{k}}^{\prime}=0$ where $a_{i}$ and $a_{j}$ refer to any elements in $A$, and $b_{k}$ to any element in $B$; in short, ${ }_{a a} R_{b}^{\prime}=0$. By an analogous argument we find that ${ }_{c c} R_{b}^{\prime}=0$. These two equations in combination with the eight equations in the last two columns of (7) constitute according to Theorem I a sufficient condition for the local separability of each of the three subsets $A, B$, and $C$ of $X$ in $f(X)$. According to the lemma proved above the individual local separability of subsets $A, B$ and $C$ in set $X$ implies their simultaneous local separability. Thus it is shown that (6) follows from (4) and (5).

Now we can proceed to demonstrate that $A, B$ and $C$ represent additively locally separable subsets of the set $A \cup B \cup C$.

Let ${ }_{\alpha \beta} R$ be defined, in analogy to ${ }_{i j} R$, by

$$
{ }_{\alpha \beta} \mathrm{R}=u_{\alpha}^{\prime} / u_{\beta}^{\prime} ;
$$

then expressing the first two equations in the first column of (7) in terms of (6) we have

$$
\begin{aligned}
& { }_{a b} R_{c}^{\prime}=\frac{\alpha_{a}^{\prime}}{\beta_{b}^{\prime}}{ }_{\alpha \beta} R_{\gamma}^{\prime} \gamma_{c}^{\prime}=0, \\
& { }_{a b} R_{a}^{\prime}=\frac{\alpha_{a}^{\prime}}{\beta_{b}^{\prime}}{ }_{\alpha \beta} R_{a}^{\prime}=0 .
\end{aligned}
$$

Since the assumed properties of function $f(X)$ exclude $\left(\alpha_{a}^{\prime} / \beta_{b}^{\prime}\right) \boldsymbol{\gamma}_{c}^{\prime}=0$ and $\alpha_{a}^{\prime} / \beta_{b}^{\prime}=0$, it follows that 


$$
{ }_{\alpha \beta} R_{\gamma}^{\prime}=0 \text { and }{ }_{\alpha \beta} R_{o}^{\prime}=0 .
$$

Being thus independent of $\gamma$, and of any element $g$ of $G,{ }_{\alpha \beta} R$ can be considered to be a function of $\alpha$ and $\beta$ alone:

$$
{ }_{\alpha \beta} R={ }_{\alpha \beta} R(\alpha, \beta) .
$$

Analogous arguments show that

$$
\begin{array}{ll}
{ }_{\gamma \alpha} R_{\beta}^{\prime}=0 & \text { and } \quad{ }_{\gamma \alpha} R_{a}^{\prime}=0, \\
{ }_{\beta \gamma} R_{\alpha}^{\prime}=0 & \text { and } \quad{ }_{{ }_{\gamma}} R_{a}^{\prime}=0,
\end{array}
$$

and consequently

$$
\begin{aligned}
& { }_{\gamma \alpha} R={ }_{{ }_{\alpha}} R(\gamma, \alpha), \\
& { }_{\beta \gamma} R={ }_{\beta \gamma} R(\beta, \gamma) .
\end{aligned}
$$

Multiplying all the left-hand and all the right-hand terms of the three equations (8a), (9a), and (10a) with each other and expressing the resulting equation in logarithms we have:

$$
\begin{aligned}
\log { }_{\alpha \beta} R(\alpha, \beta)+ & \log _{\gamma \alpha} R(\gamma, \alpha)+\log { }_{\beta \gamma} R(\beta, \gamma) \\
& =\log u_{\alpha}^{\prime} / u_{\beta}^{\prime}+\log u_{\gamma}^{\prime} / u_{\alpha}^{\prime}+\log u_{\beta}^{\prime} / u_{\gamma}^{\prime}=\log 1=0 .
\end{aligned}
$$

If ${ }_{\alpha \beta} R(\alpha, \beta),{ }_{\gamma \alpha} R(\gamma, \alpha)$ or ${ }_{\beta \gamma} R(\beta, \gamma)$ is negative, they can be made positive by temporarily replacing one of the variables $\alpha, \beta$, or $\gamma$ by its negative. An opposite substitution must then be made at the later stage of the argument after natural numbers have again been substituted for logarithms. Successive partial differentiation of both sides of the last equation with respect to $\alpha$ and $\beta$ gives

$$
\frac{\partial^{2} \log _{\alpha \beta} R(\alpha, \beta)}{\partial \alpha \partial \beta}=0,
$$

which means that $\log _{\alpha \beta} R(\alpha, \beta)$ can be written as a sum of two terms, one of which is a function of $\alpha$ alone and the other a function of $\beta$ alone:

$$
\log _{\alpha \beta} R(\alpha, \beta)=\log \lambda(\alpha)+\log \mu(\beta)
$$

or

$$
u_{\alpha}^{\prime} / u_{\beta}^{\prime}=\lambda(\alpha) \mu(\beta) .
$$

The characteristic equations of this partial differential equation are

$$
\frac{d \alpha}{1 / \lambda(\alpha)}=\frac{d \beta}{-\mu(\beta)}=\frac{d u}{0}=\frac{d \gamma}{0}=\frac{d x_{k}}{0}=\cdots=\frac{d x_{n}}{0},
$$


where $x_{k}, \cdots, x_{n}$ are variables in $G$ and its general integral is

$$
r(\alpha)+t(\beta)+m(\gamma, G, y)=0,
$$

where

$$
y=f(X)=u(\alpha, \beta, \gamma, G) .
$$

Expressing the two equations in (9) in terms of (11) and in accordance with the definition of ${ }_{\alpha \gamma} R$ and using implicit differentiation to find the partial derivatives of $u$, we have,

$$
\begin{aligned}
{ }_{\alpha \gamma} R_{\beta}^{\prime} & =\frac{\partial}{\partial \beta}\left(\frac{r^{\prime}(\alpha)}{m_{\gamma}^{\prime}(\gamma, G, y)}\right)=-\frac{r^{\prime}(\alpha) m_{\gamma y}^{\prime \prime}(\gamma, G, y) u_{\beta}^{\prime}}{\left[m_{\gamma}^{\prime}(\gamma, G, y)\right]^{2}}=0, \\
{ }_{\alpha \gamma} R_{\theta}^{\prime} & =\frac{\partial}{\partial g}\left(\frac{r^{\prime}(\alpha)}{m_{\gamma}^{\prime}(\gamma, G, y)}\right) \\
& =-\frac{r^{\prime}(\alpha)\left[m_{\gamma \nu}^{\prime \prime}(\gamma, G, y) u_{g}^{\prime}+m_{\gamma \theta}^{\prime \prime}(\gamma, G, y)\right]}{\left[m_{\gamma}^{\prime}(\gamma, G, y)\right]^{2}}=0 .
\end{aligned}
$$

In the operations of partial differentiation indicated by the middle terms of the two expressions, $\alpha$ and $\gamma$ in accordance with Theorem I are held constant; in the first expression $y$ and $\beta$, in the second $y$ and $g$, are allowed to vary. The partial derivative $m_{\gamma}^{\prime}(\gamma, G, y)$ is by assumption different from zero. Since no other functions in the third terms of these two expressions can vanish,

$$
\begin{gathered}
m_{\gamma y}^{\prime \prime}(\gamma, G, y)=0, \\
m_{\gamma y}^{\prime \prime}(\gamma, G, y) u_{g}^{\prime}+m_{\gamma \theta}^{\prime \prime}(\gamma, G, y)=0,
\end{gathered}
$$

from which

$$
m_{\gamma \theta}^{\prime \prime}(\gamma, G, y)=0 .
$$

These equations imply that function $m$ has the general form

$$
m(\gamma, G, y)=K(\gamma)+l(G, y) .
$$

Substitution in (11) gives

$$
r(\alpha)+t(\beta)+K(\gamma)+l(G, y)=0
$$

and solving for $y$, we obtain

$$
y=h(r(\alpha)+t(\beta)+K(\gamma), G) .
$$

But $\alpha$ is a function of $A, \beta$ is a function of $B$, and $\gamma$ a function of $C$; thus the same relationship can be written as 


$$
y=f(X)=u(\alpha(A)+\beta(B)+\gamma(C), G),
$$

which shows that:

i. The function $\alpha(A)+\beta(B)+\gamma(C)$ of the set $A \cup B \cup C$ is locally separable in $f(X)$ and this set itself is a locally functionally separable subset of $X$ in $f(X)$.

ii. The sets $A, B$ and $C$ are additively locally separable subsets of $A \cup B \cup C$ in $\alpha(A)+\beta(B)+\gamma(C)$.

HARVARD UNIVERSITY 